\title{
Concentration effects on photophoresis of aerosol spheres
}

\author{
Huan J. Keh *, Yu S. Chen \\ Department of Chemical Engineering, National Taiwan University, Taipei 106-17, Taiwan, ROC
}

Received 2 March 2001; accepted 25 June 2001

\begin{abstract}
The photophoretic motion of a homogeneous suspension of identical spherical particles of arbitrary thermal conductivity and surface properties exposed to a radiative flux is considered under conditions of small Knudsen, Reynolds, and Peclet numbers. The effects of interaction of the individual particles are taken into explicit account by employing a unit cell model which is known to provide good predictions for the sedimentation of monodisperse suspensions of spherical particles. The appropriate equations of conservation of energy and momentum are solved for each cell, in which a spherical particle is envisaged to be surrounded by a concentric shell of suspending fluid, and the photophoretic velocity of the particle is calculated for various cases. Analytical expressions of this mean particle velocity are obtained in closed form as monotonically decreasing functions of the non-vanishing volume fraction of the particles. The normalized photophoretic velocity increases with an increase in the relative conductivity of the particles for a given particle concentration. (C) 2002 Elsevier Science B.V. All rights reserved.
\end{abstract}

Keywords: Photophoresis of aerosol spheres; Effects of particle volume fraction; Unit cell model

\section{Introduction}

When a small suspended particle in a gaseous medium is exposed to an intense light beam, it will spontaneously migrate parallel to the direction of the light. This phenomenon, which is a direct result of the uneven heating of the light absorbing particle (and therefore, of its adjacent gas molecules), was termed photophoresis by its discoverer, Ehrenhaft, around 1910 [1,2]. The photophoretic (or thermophoretic) effect can be explained in part by appealing to the kinetic

\footnotetext{
* Corresponding author. Tel.: + 886-2-2363-5462; fax: + 886-2-2362-3040.

E-mail address: huan@ccms.ntu.edu.tw (H.J. Keh).
}

theory of gases [3,4]. The higher-energy molecules in the hot region of the gas impinge on the particle with greater momenta than molecules coming from the cold region, thereby leading to the migration of the particle in the direction opposite to the surface temperature gradient. Thus, the photophoretic force on an aerosol particle can be directed either toward (negative photophoresis) or away from (positive photophoresis) the light source, depending upon the optical characteristics of the particle. If the particle is opaque and the incident light energy is absorbed and dissipated directly at the front surface of the particle, positive photophoresis occurs. Conversely, if the light beam is partially transmitted and focused at some other internal area (e.g. the rear surface) of the particle, motion in other directions may result. 
Since photophoresis is observed for many particulate materials in the diameter range between $10^{-8}$ and $10^{-3} \mathrm{~m}$, and at pressures from above $1 \mathrm{~atm}$ down to below 1 torr, under illumination intensities comparable with sunlight [2], the results of photophoresis investigations are of interest to a wide variety of fields including cloud physics, aerosol science, and environmental engineering. For example, measurements of the photophoretic force or the reversal point from positive to negative photophoresis with the elaboration of photophoretic spectroscopy can be used to determine the physical properties, such as the complex refractive index, and the chemical composition of aerosol particles [5]. The photophoretic phenomena of aerosol particles subjected to coherent light beams have been applied to the development of laser atmospheric monitoring methods [6]. It was found that, due to the effect of both positive and negative photophoresis, stratospheric aerosol particles may be caused to rise against gravity while others are induced to fall considerably more rapidly than they would under gravity alone [7]. Considering that radiative transfer can account for approximately $95 \%$ of the total heat flux in pulverized-coal furnaces, the driving force for photophoresis of small particles in combustion environments can be significantly greater than that for thermophoresis [8].

On the basis of the assumptions of small Knudsen number $(l / a$, where $a$ is the radius of the particle and $l$ the mean free path of the surrounding gas molecules), small Peclet number, and small Reynolds number, as well as the effects of temperature jump, thermal slip, and frictional slip at the gas-particle interface, Reed [9] and Mackowski [8] obtained the photophoretic velocity of an aerosol sphere illuminated by an intense light beam as

$U^{(0)}=\frac{2 C_{\mathrm{s}} J_{1} \eta I}{3\left(1+2 C_{\mathrm{m}} l / a\right)\left(2 k+k_{\mathrm{p}}+2 k_{\mathrm{p}} C_{\mathrm{t}} l / a\right) \rho T_{0}}$

Here, $I$ is the intensity of the incident light (incoming illumination energy flux); $\rho, \eta$, and $k$ are the density, viscosity, and thermal conductivity, respectively, of the gas; $k_{\mathrm{p}}$ is the thermal conductivity of the particle; $T_{0}$ is the absolute temperature of the bulk gas; $J_{1}$ is the so-called photophoretic asymmetry factor [10] defined by Eq. (26), which can be either positive (negative photophoresis) or negative (positive photophoresis); $C_{\mathrm{s}}, C_{\mathrm{t}}$, and $C_{\mathrm{m}}$ are dimensionless coefficients accounting for the thermal slip, temperature jump, and hydrodynamic slip phenomena, respectively, at the particle surface and must be determined experimentally for each gas-solid system. A set of reasonable kinetic-theory values for complete thermal and momentum accommodations appear to be $C_{\mathrm{s}}=1.17, C_{\mathrm{t}}=2.18$, and $C_{\mathrm{m}}=1.14$ [11].

In practical applications of photophoresis, collections of aerosol particles are usually encountered, and effects of particle interactions will be important. However, the effect of particle concentration on the photophoretic mobility for a suspension of aerosol particles has not been investigated yet. A unit cell model has been employed successfully (and tested against the experimental data) to predict the effect of particle concentration on the mean sedimentation rate in a bounded suspension of identical spherical particles [12-16]. This model involves the concept that an assemblage can be divided into a number of identical cells, one sphere occupying each cell at its center. The boundary-value problem for multiple spheres is thus reduced to the consideration of the behavior of a single sphere and its bounding envelope. Although different shapes of cells can be employed, the assumption of a spherical shape for the fictitious envelope of suspending fluid surrounding each spherical particle is of great convenience. Although the Brownian motion of small particles is not included in its analysis, the cell model can be applied in some stochastic sense to random particle arrays. In this work, the cell model is used to describe the thermal and hydrodynamic interactions among aerosol spheres in a monodisperse suspension subjected to an intense light beam. The analytical solution in a closed form obtained with this model enables the average photophoretic velocity to be predicted as functions of the volume fraction of the particles for various cases. 


\section{Analysis}

We consider the steady photophoretic motion of a uniform three-dimensional distribution of identical spherical particles of arbitrary thermal conductivity and surface properties in a gaseous medium. The incident light is imposed in the $z$ direction with intensity (incoming illumination energy flux) $I$ and the photophoretic velocity of the particles is $U e_{z}$, where $e_{z}$ is the unit vector in the positive $z$ direction. As shown in Fig. 1, we employ a unit cell model in which each particle of radius $a$ is surrounded by a concentric spherical shell of suspending fluid having an outer radius of $b$ such that the particle/cell volume ratio is equal to the particle volume fraction $\varphi$ throughout the entire suspension; namely, $\varphi=(a / b)^{3}$. The Knudsen numbers $l / a$ and $l /(b-a)$ are assumed to be small so that the fluid flow is in the continuum regime and the Knudsen layers surrounding the particles do not overlap with one another. The origin of the spherical coordinate system $(r, \theta, \phi)$ is set at the center of the particle. Our objective is to determine the particle velocity $U$ in a cell induced by the photophoretic (radiometric) driving force.

The Peclet number of this axisymmetric problem is assumed to be small. Hence, the equation of energy governing the temperature distribution $T(r, \theta)$ for the fluid of constant thermal conductivity $k$ is the Laplace equation,

$$
\nabla^{2} T=0 \quad(a \leq r \leq b) .
$$

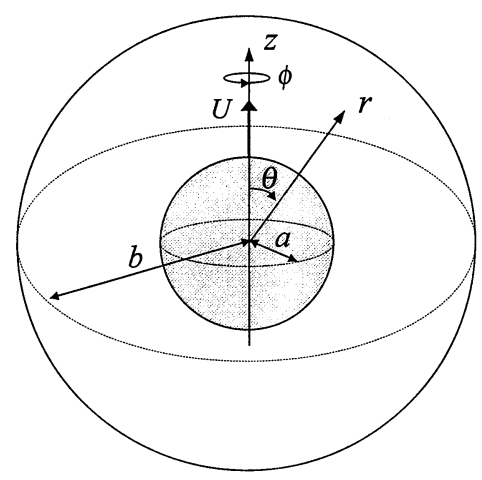

Fig. 1. Geometrical sketch for the photophoretic motion of a spherical particle at the center of a spherical cell.
The temperature distribution $T_{\mathrm{p}}(r, \theta)$ inside the radiation-absorbing particle is described by

$\nabla^{2} T_{\mathrm{p}}=-\frac{1}{k_{\mathrm{p}}} Q(r, \theta) \quad(r \leq a)$,

where $k_{\mathrm{p}}$ is the thermal conductivity of the particle and $Q(r, \theta)$ is the volumetric thermal energy generation rate resulting from local radiation absorption. For a plane monochromatic wave, the source function $Q(r, \theta)$ is related to the electric field $\mathbf{E}(r, \theta)$ inside the particle according to the Lorenz-Mie theory $[8,17]$,

$Q(r, \theta)=\frac{4 \pi v \kappa I}{\lambda} \frac{|\mathbf{E}(r, \theta)|^{2}}{\left|\mathbf{E}_{0}\right|^{2}}=\frac{4 \pi v \kappa I}{\lambda} B(\zeta, \theta)$.

Here, $v$ and $\kappa$ are the real and imaginary parts of the complex refractive index $N(N=v+i \kappa)$ of the particle, $\mathbf{E}_{0}$ is the incident electric field strength, $\lambda$ is the wavelength of the incident radiation, $B(\zeta, \theta)$ is the dimensionless electric field distribution function, and $\zeta=r / a$.

The boundary conditions at the particle surface $(r=a)$ require that the normal heat fluxes be continuous and a temperature jump which is proportional to the normal temperature gradient [4] occur. Also, the fluid temperature at the outer (virtual) surface of the cell approaches the bulkgas temperature $T_{0}$ which is a constant. Thus,

$r=a$ :

$k \frac{\partial T}{\partial r}=k_{\mathrm{p}} \frac{\partial T_{\mathrm{p}}}{\partial r}, \quad T-T_{\mathrm{p}}=C_{\mathrm{t}} l \frac{\partial T}{\partial r}$,

$r=0: \quad T_{\mathrm{p}}$ is finite,

$r=b: \quad T=T_{0}$,

where $C_{\mathrm{t}}$ is the temperature jump coefficient about the particle surface. A sufficiently general solution to Eqs. (2) and (3) is

$$
\begin{aligned}
& T=T_{0}+\frac{a I}{k} \sum_{n=0}^{\infty} A_{n}\left[\zeta^{-n-1}-\varphi^{(2 n+1) / 3} \zeta\right] P_{n}(\cos \theta), \\
& T_{\mathrm{p}}=T_{0}+\frac{a I}{k_{\mathrm{p}} n=0} \sum_{n}^{\infty}\left[B_{n} \zeta^{n}+S_{n}(\zeta)\right] P_{n}(\cos \theta),
\end{aligned}
$$

where 
$S_{n}(\zeta)=\frac{2 \pi v \kappa a}{\lambda}\left[\zeta^{n} \int_{\zeta}^{1} t^{-n+1} \int_{0}^{\pi} B(t, \theta) P_{n}(\cos \theta)\right.$

$\sin \theta \mathrm{d} \theta \mathrm{d} t+\zeta^{-n-1} \int_{0}^{\zeta} t^{n+2}$

$\left.\int_{0}^{\pi} B(t, \theta) P_{n}(\cos \theta) \sin \theta \mathrm{d} \theta \mathrm{d} t\right]$,

$P_{n}$ is the Legendre polynomial of order $n$, and $\varphi=(a / b)^{3}$. A solution of this form immediately satisfies boundary conditions (6) and (7). The unknown coefficients $A_{n}$ and $B_{n}$ are to be determined using the boundary conditions at the particle surface.

Applying the boundary conditions [Eqs. (5a) and (5b)] at the surface of the particle to Eqs. (8) and (9), we obtain

$$
\begin{aligned}
& A_{n}= \\
& \frac{n S_{n}(1)-S_{n}^{\prime}(1)}{n k^{*}+(n+1)\left(1+n k^{*} C_{\mathrm{t}}^{*}\right)+n\left(1-k^{*}+k^{*} C_{\mathrm{t}}^{*}\right) \varphi^{(2 n+1) / 3}},
\end{aligned}
$$

is given by

$$
E^{2}=\frac{\partial^{2}}{\partial r^{2}}+\frac{\sin \theta}{r^{2}} \frac{\partial}{\partial \theta}\left(\frac{1}{\sin \theta} \frac{\partial}{\partial \theta}\right) .
$$

The stream function $\Psi$ is related to the $r$ and $\theta$ components of the velocity field $\mathbf{v}$ by

$$
\begin{aligned}
& v_{\mathrm{r}}=-\frac{1}{r^{2} \sin \theta} \frac{\partial \Psi}{\partial \theta}, \\
& v_{\theta}=\frac{1}{r \sin \theta} \frac{\partial \Psi}{\partial r} .
\end{aligned}
$$

Owing to the thermal and frictional slip velocities along the solid-fluid interface, the boundary conditions for the fluid velocity at the particle surface are [18]

$$
\begin{aligned}
& r=a: \\
& v_{\mathrm{r}}=U \cos \theta,
\end{aligned}
$$

where

$k^{*}=k_{\mathrm{p}} / k$,

$C_{\mathrm{t}}^{*}=C_{\mathrm{t}} l / a$,

and the prime on $S_{n}(\zeta)$ means differentiation with respect to $\zeta$. Note that the dimensionless parameter $k^{*} C_{\mathrm{t}}^{*}$ denotes the relative resistance caused by the temperature jump at the particle surface with respect to the heat conduction inside the particle.

With knowledge of the solution for the temperature distribution, we can now proceed to find the flow field in a cell. The fluid surrounding the particle is assumed to be incompressible and Newtonian. Due to the low Reynolds number, the fluid motion caused by the photophoretic migration of the particle is governed by the quasisteady fourth-order differential equation for axisymmetric creeping flows,

$E^{4} \Psi=E^{2}\left(E^{2} \Psi\right)=0$,

where $\Psi(r, \theta)$ is the Stokes stream function. In spherical coordinates, the Stokesian operator $E^{2}$
$v_{\theta}=-U \sin \theta+\frac{C_{m} l}{\eta} \tau_{\mathrm{r} \theta}+\frac{C_{\mathrm{s}} \eta}{\rho T_{0}} \frac{\partial T}{r \partial \theta}$.

Here, $C_{\mathrm{m}}$ and $C_{\mathrm{s}}$ are the frictional and thermal slip coefficients, respectively, about the surface of the particle, $\tau_{r \theta}$ is the shear stress for the fluid flow,

$\tau_{r \theta}=\eta\left[r \frac{\partial}{\partial r}\left(\frac{v_{\theta}}{r}\right)+\frac{1}{r} \frac{\partial v_{\mathrm{r}}}{\partial \theta}\right]$,

and $U$ is the photophoretic velocity of the particle to be determined. The derivative $\partial T / \partial \theta$ along the particle surface can be evaluated from the temperature distribution given by Eq. (8). The validity of the expression for the thermal slip velocity in Eq. (17b) is based on the assumption that the fluid temperature is only slightly non-uniform on the length scale of the particle radius. Note that the slip-flow boundary condition is not only applicable in the continuum regime $(l / a \ll 1)$, but also appears to be valid for some cases even into the molecular flow regime $(l / a \geq 1)$. Generally speak- 
ing, the slip condition becomes increasingly more important for small particles. On the outer boundary of the cell, the Happel model [12] assumes that the radial velocity and the shear stress are zero; namely,

$r=b: \quad v_{\mathrm{r}}=0$,

$\tau_{\mathrm{r} \theta}=0$.

A solution to Eq. (14) suitable for satisfying boundary conditions on the spherical surfaces is [19]

$\Psi=\left(C r^{-1}+D r+E r^{2}+F r^{2}\right) \sin ^{2} \theta$,

where the constants $C, D, E$, and $F$ are to be determined from Eqs. (17a), (17b), (19a) and (19b) using Eqs. (8), (16a) and (16b). The procedure is straightforward, with the result

$$
\begin{aligned}
C= & a^{3} \omega\left[U+3 A_{1}\left(1-\varphi^{1 / 3}\right) V\right], \\
D= & -a \omega\left\{\left[3\left(1+2 C_{\mathrm{m}}^{*}\right)+2\left(1-3 C_{\mathrm{m}}^{*}\right) \varphi^{5 / 3}\right]\right. \\
& \left.U+3 A_{1}\left(1-\varphi^{5 / 3}\right) V\right\}, \\
E= & \omega\left\{\left[3\left(1+2 C_{\mathrm{m}}^{*}\right) \varphi^{1 / 3}\right.\right. \\
& \left.\left.+2\left(1-3 C_{\mathrm{m}}^{*}\right) \varphi^{2}\right] U+3 A_{1}\left(\varphi^{1 / 3}-\varphi^{2}\right) V\right\}, \\
F= & -a^{-2} \omega\left[\varphi^{5 / 3} U+3 A_{1}\left(\varphi^{5 / 3}-\varphi^{2}\right) V\right],
\end{aligned}
$$

where

$$
\begin{aligned}
& C_{\mathrm{m}}^{*}=C_{\mathrm{m}} l / a, \\
& V=\frac{2 C_{\mathrm{s}} \eta I}{3 k \rho T_{0}}(1-\varphi),
\end{aligned}
$$

which is a characteristic migration velocity of the particle, and

$$
\begin{aligned}
\omega= & {\left[2\left(2-3 \varphi^{1 / 3}+3 \varphi^{5 / 3}-2 \varphi^{2}\right)\right.} \\
& \left.+12 C_{\mathrm{m}}^{*}\left(1-\varphi^{1 / 3}-\varphi^{5 / 3}+\varphi^{2}\right)\right]^{-1} .
\end{aligned}
$$

With this solution, the components of the fluid velocity in this axisymmetric flow (with $v_{\phi}=0$ ) can be calculated by using Eqs. (16a) and (16b).

The coefficient $A_{1}$ in Eqs. (21a), (21b), (21c) and (21d) can be calculated from the definition of Eq. (11),

$$
A_{1}=\frac{J_{1}}{2+k^{*}+2 k^{*} C_{\mathrm{t}}^{*}+\left(1-k^{*}+k^{*} C_{\mathrm{t}}^{*}\right) \varphi},
$$

where $J_{1}$ is the so-called photophoretic asymmetry factor,

$J_{1}=\frac{6 \pi v \kappa a}{\lambda} \int_{0}^{1} \int_{0}^{\pi} B(\zeta, \theta) \zeta^{3} \cos \theta \sin \theta \mathrm{d} \theta \mathrm{d} \zeta$,

which depends on the complex refractive index $(N=v+i \kappa)$ and the normalized size $(2 \pi a / \lambda)$ of the particle. The asymmetry factor represents a weighted integration of the heat source function over the particle volume and defines the sign and the magnitude of the photophoretic force. If $J_{1}<$ 0 , the particle moves in the direction of the light beam (positive photophoresis). If $J_{1}>0$, the particle moves in the opposite direction (negative photophoresis). For a completely opaque spherical particle the heat sources are concentrated on the illuminated part of the particle surface, namely,

$$
B(\zeta, \theta)=\left\{\begin{array}{cc}
\frac{-\lambda}{2 \pi v \kappa a} \cos \theta \delta(\zeta-1) & \text { for } \frac{\pi}{2} \leq \theta \leq \pi \\
0 & 0 \leq \theta \leq \frac{\pi}{2},
\end{array}\right.
$$

where $\delta(\zeta-1)$ is a Dirac delta function which equals infinity if $\zeta=1$ and vanishes otherwise. The substitution of Eq. (27) into Eq. (26) results in $J_{1}=-1 / 2$ knowing that $\int_{0}^{1} g(\zeta) \delta(\zeta-1) \mathrm{d} \zeta=(1 /$ 2) $g(1)$. It is obvious that the range of the asymmetry factor is $-1 / 2 \leq J_{1} \leq 1 / 2$. According to Eq. (1), the photophoretic velocity at illumination of an intensity comparable with the solar constant $\left(1353 \mathrm{~W} \mathrm{~m}^{-2}\right)$ is of the order of $10^{-5} \mathrm{~m} \mathrm{~s}^{-1}$.

The drag force (in the $z$ direction) exerted by the fluid on the particle is [19]

$F_{\mathrm{d}}=8 \pi \eta D$.

Because the particle is freely suspended in the fluid, the net force exerted by the fluid on the particle must vanish; namely, $D=0$. With this constraint, Eq. (21b) yields the photophoretic velocity of the particle,

$$
\begin{aligned}
U= & -3 A_{1}\left(1-\varphi^{5 / 3}\right) \\
& \times\left[3\left(1+2 C_{\mathrm{m}}^{*}\right)+2\left(1-3 C_{\mathrm{m}}^{*}\right) \varphi^{5 / 3}\right]^{-1} V .
\end{aligned}
$$

If the Kuwabara model [13] for the boundary conditions of the fluid flow at the virtual surface of the cell, which assumes that the radial velocity and the vorticity are zero, is used, Eq. (19b) is replaced by 
$r=b: \quad(\nabla \times \mathbf{v})_{\phi}=\frac{\partial v_{\theta}}{\partial r}+\frac{v_{\theta}}{r}-\frac{1}{r} \frac{\partial v_{\mathrm{r}}}{\partial \theta}=0$.

With this change, the stream function $\Psi$ can still be expressed in the form of Eq. (20), and the coefficients $C, D, E$, and $F$ should be determined by boundary conditions (17), (19a), and (30). The result is

$$
\begin{aligned}
C= & a^{3} \omega^{\prime}\left[\left(5-2 \varphi+6 C_{\mathrm{m}}^{*} \varphi\right) U\right. \\
& \left.+3 A_{1}\left(5-6 \varphi^{1 / 3}+\varphi\right) V\right], \\
D= & -15 a \omega^{\prime}\left[\left(1+2 C_{\mathrm{m}}^{*}\right) U+A_{1}(1-\varphi) V\right], \\
E= & \omega^{\prime}\left\{\left[18 \varphi^{1 / 3}-5 \varphi+2 \varphi^{2}+6 C_{\mathrm{m}}^{*}\left(6 \varphi^{1 / 3}\right.\right.\right. \\
& \left.\left.\left.-\varphi^{2}\right)\right] U+3 A_{1}\left(6 \varphi^{1 / 3}-5 \varphi-\varphi^{2}\right) V\right\}, \\
F= & -3 a^{-2} \omega^{\prime}\left[\left(1+2 C_{m}^{*}\right) \varphi U+A_{1} \varphi(1-\varphi) V\right],
\end{aligned}
$$

where

$$
\begin{aligned}
\omega^{\prime}= & {\left[4\left(5-9 \varphi^{1 / 3}+5 \varphi-\varphi^{2}\right)\right.} \\
& \left.+12 C_{\mathrm{m}}^{*}\left(5-6 \varphi^{1 / 3}+\varphi^{2}\right)\right]^{-1} .
\end{aligned}
$$

The fact that there is no drag force exerted on the particle requires $D=0$, and Eq. (31b) gives the particle velocity as

$$
U=-A_{1}(1-\varphi)\left(1+2 C_{\mathrm{m}}^{*}\right)^{-1} V .
$$

For the sedimentation of a suspension of identical spherical particles, both the Happel and the Kuwabara models give qualitatively the same flow fields and approximately comparable drag forces. However, the Happel model has an advantage in that it does not require an exchange of mechanical energy between the cell and the environment [19]. For photophoresis, the Kuwabara model might be a better choice owing to the fact that its boundary condition of zero vorticity at the virtual surface of the cell is consistent with the irrotational-flow environment generated by a photophoretic particle [20].

\section{Results and discussion}

The analytical solutions of the temperature and flow fields in the unit cell and of the photophoretic velocity of the particle have been ob- tained in the previous section for both the Happel model and the Kuwabara model.

For the Happel model, the particle velocity is given by Eq. (29). This migration velocity can be expressed as

$$
\begin{aligned}
U= & U^{(0)}(1-\varphi)\left(1-\varphi^{5 / 3}\right)(1+G \varphi)^{-1} \\
& \times\left[1+\frac{2\left(1-3 C_{\mathrm{m}}^{*}\right)}{3\left(1+2 C_{\mathrm{m}}^{*}\right)} \varphi^{5 / 3}\right]^{-1},
\end{aligned}
$$

where

$$
U=U^{(0)}=-\frac{2 C_{\mathrm{s}} J_{1} \eta I}{3\left(1+2 C_{\mathrm{m}}^{*}\right)\left(2+k^{*}+2 k^{*} C_{\mathrm{t}}^{*}\right) k \rho T_{0}},
$$

which is the photophoretic velocity of the particle given by Eq. (1) in the limit $\varphi=0$, and

$$
G=\frac{1-k^{*}+k^{*} C_{\mathrm{t}}^{*}}{2+k^{*}+2 k^{*} C_{\mathrm{t}}^{*}} .
$$

It can be found that $-1 \leq G \leq 1 / 2$. For the case of large particle $(l / a \ll 1)$ with $k^{*} \gg 1, G \rightarrow-1$. For the case of $k^{*} \ll 1, G \rightarrow 1 / 2$. When $k^{*}=$ $\left(1-C_{\mathrm{t}}^{*}\right)^{-1}, G=0$.

The particle velocity $U$ can also be written as the following power expansion in $\varphi$ :

$$
U=U^{(0)}\left[1+\alpha \varphi+\beta \varphi^{5 / 3}+O\left(\varphi^{2}\right)\right] .
$$

A comparison between Eqs. (34) and (37) leads to the coefficients $\alpha$ and $\beta$ for the Happel model,

$$
\begin{aligned}
& \alpha=-1-G, \\
& \beta=-\frac{5}{3\left(1+2 C_{\mathrm{m}}^{*}\right)} .
\end{aligned}
$$

Note that both $\alpha$ and $\beta$ are negative. Also, $\alpha$ is only related to the parameters $k^{*}$ ( $\alpha$ is a monotonically increasing function of $\left.k^{*}\right)$ and $C_{\mathrm{t}}^{*}$, and $\beta$ is a function of the parameter $C_{\mathrm{m}}^{*}$ only $(\beta$ is a monotonically increasing function of $C_{\mathrm{m}}^{*}$ ).

For the Kuwabara model, the photophoretic velocity of the particle is given by Eq. (33), which can be written as

$U=U^{(0)}(1-\varphi)^{2}(1+G \varphi)^{-1}$.

This particle velocity can also be expressed in the expansion form of Eq. (37), with the coefficients $\alpha$ and $\beta$ given by 
$\alpha=-2-G$,

$\beta=0$.

Note that $\alpha$ in this case equals the value of $\alpha$ in the Happel model -1 .

It can be found from Eqs. (34) and (39) that the mean photophoretic velocity in a homogeneous suspension of identical aerosol spheres predicted by the cell model depends upon the boundary conditions specified at the virtual surface of the cell. The boundary condition for the temperature at the virtual surface $r=b$ determines the dependence of the normalized particle velocity (or mobility) $U / U^{(0)}$ as a function of $k^{*}$ and $C_{\mathrm{t}}^{*}$, while the boundary condition for the fluid velocity at $r=b$ controls the connection of $U / U^{(0)}$ with $C_{\mathrm{m}}^{*}$. Interestingly, the Kuwabara model predicts that $U / U^{(0)}$ is independent of the value of $C_{\mathrm{m}}^{*}$. For given values of $k^{*}, C_{\mathrm{t}}^{*}, C_{\mathrm{m}}^{*}$, and $\varphi$, the mean particle velocity obtained from the Kuwabara model is always smaller than those obtained from the Happel model.

The normalized photophoretic velocity in a homogeneous suspension of identical aerosol spheres, $U / U^{(0)}$, as calculated from Eqs. (34) and (39) for the two cases of the cell model, is plotted versus the volume fraction of the particles, $\varphi$, in Figs. 2-4 for various values of the parameters $k^{*}$, $C_{\mathrm{t}}^{*}$, and $C_{\mathrm{m}}^{*}$. In both cases, $U / U^{(0)}$ equals unity in the limit $\varphi=0$ and decreases monotonically with an increase in $\varphi$ for any given values of $k^{*}, C_{\mathrm{t}}^{*}$, and $C_{\mathrm{m}}^{*}$. The calculations are presented up to $\varphi=0.74$, which corresponds to the maximum attainable volume fraction for a swarm of identical spheres [21]. It is clear that at volume fractions approaching this, coagulation due to contacts between particles may dominate, and the present study with the assumption of monodispersity and sphericity does not cover this case.

Fig. 2 shows the results of $U / U^{(0)}$ as a function of $\varphi$ for a suspension of identical particles with $k^{*}=\left(1-C_{\mathrm{t}}^{*}\right)^{-1}($ or $G=0)$ for the Happel model with $C_{\mathrm{m}}^{*}$ as a parameter. The corresponding results for the Kuwabara model, which are independent of $C_{\mathrm{m}}^{*}$, are also plotted in this figure by a dashed curve for comparison. It can be seen that $U / U^{(0)}$ obtained from the Happel model is a monotonically increasing function of $C_{\mathrm{t}}^{*}$ for a

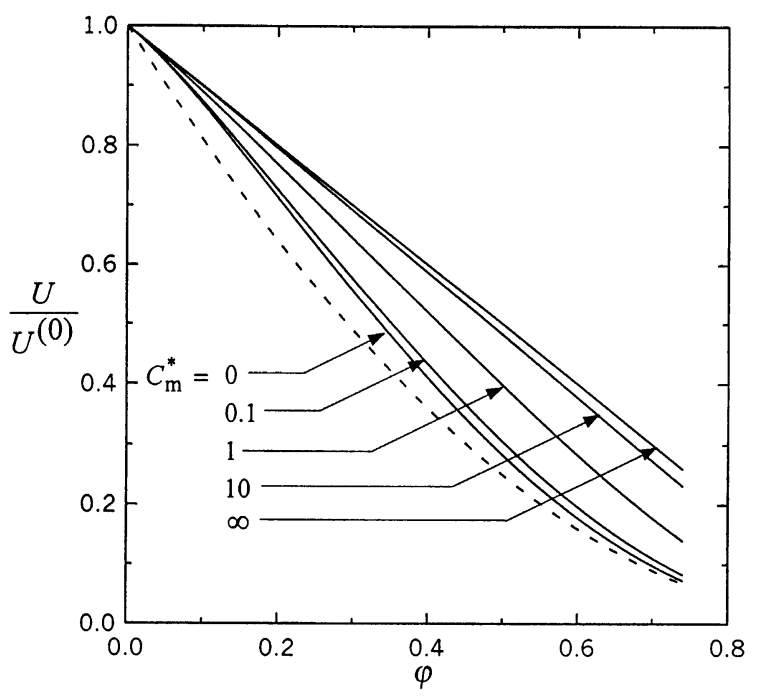

Fig. 2. Plots of the normalized photophoretic velocity in a monodisperse suspension of spherical particles with $k^{*}=(1-$ $\left.C_{\mathrm{t}}^{*}\right)^{-1}$ versus the volume fraction of the particles with $C_{\mathrm{m}}^{*}$ as a parameter for the Happel model (Eq. (34)). The dashed curve represents the case of the Kuwabara model (Eq. (39)) for comparison.

fixed value of $\varphi$. Examining Eq. (34), one can find that this tendency between $U / U^{(0)}$ and $C_{\mathrm{t}}^{*}$ also exists for all values of $k^{*}$ other than $\left(1-C_{\mathrm{t}}^{*}\right)^{-1}$. When the Knudsen number is small (or $C_{\mathrm{m}}^{*}<$ 0.1 , the difference in the results of $U / U^{(0)}$ predicted by the two cell models is small. Although the calculations in Fig. 2 are presented up to the limit of infinite $C_{\mathrm{t}}^{*}$ for completeness, it is understood that the Knudsen number is large and the continuum model for the gas phase with a slipflow boundary condition at the particle surface may not be valid for this limit.

The cell-model solutions for a parallel problem concerning the sedimentation of a suspension of identical aerosol spheres were presented by Keh and Ho [16]. Similar to the case of the Happel model in photophoresis, the normalized particle mobilities predicted by both the Happel and Kuwabara models in sedimentation also increase monotonically with an increase in $C_{\mathrm{m}}^{*}$ for a constant value of $\varphi$. However, the effect of particle concentration on photophoresis is much weaker than that on sedimentation. The leading order of this effect is $\varphi$ in Eq. (37) for photophoretic 


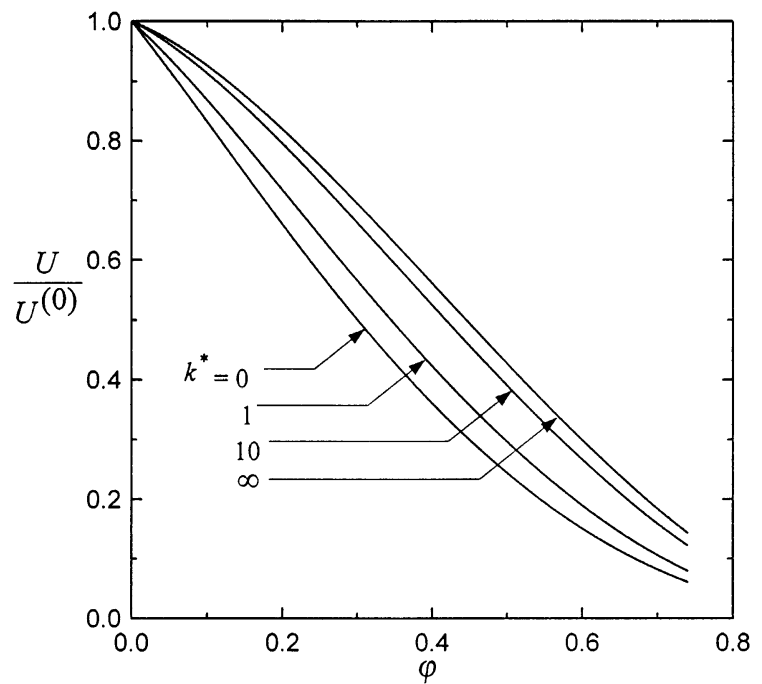

Fig. 3. Plots of the normalized photophoretic velocity in a monodisperse suspension of spherical particles with $C_{\mathrm{t}}^{*}=$ $2 C_{\mathrm{m}}^{*}=0.2$ versus the volume fraction of the particles with $k^{*}$ as a parameter for the Happel model (Eq. (34)).

motion, compared with the effect of order $\varphi^{1 / 3}$ for sedimentation. The reason for the weaker concentration effect on photophoresis is that the disturbance to the fluid velocity field caused by a

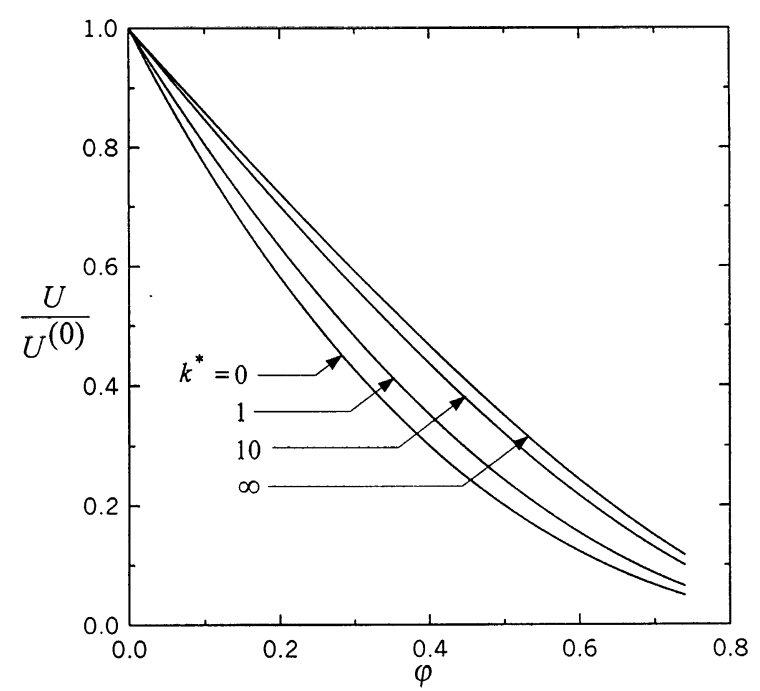

Fig. 4. Plots of the normalized photophoretic velocity in a monodisperse suspension of spherical particles with $C_{\mathrm{t}}^{*}=0.2$ versus the volume fraction of the particles with $k^{*}$ as a parameter for the Kuwabara model (Eq. (39)). photophoretic (or thermophoretic) particle decays faster (as $r^{-3}$ ) than that caused by an aerosol particle moving under the influence of a body force (as $r^{-1}$ ) [22].

The results of $U / U^{(0)}$ as a function of $\varphi$ for a monodisperse suspension of particles with $C_{\mathrm{t}}^{*}=$ $2 C_{\mathrm{m}}^{*}=0.2$ (a typical representative case) are plotted in Figs. 3 and 4 for the Happel and Kuwabara cell models, respectively, with the relative thermal conductivity $k^{*}$ of the particles as a parameter. For a given value of $\varphi, U / U^{(0)}$ increases monotonically with an increase in $k^{*}$ for both models. Examining Eqs. (34) and (39), one can find that this trend of dependence of $U / U^{(0)}$ on $k^{*}$ also exists for any other values of $C_{\mathrm{t}}^{*}$ and $C_{\mathrm{m}}^{*}$. Note that, because a relatively high conductivity of the particle reduces the local temperature gradient (and thus, the thermal slip effect) along the particle surface, the photophoretic velocity of an isolated particle, $U^{(0)}$, is a monotonically decreasing function of $k^{*}$ (and of $C_{\mathrm{t}}^{*}$ and $C_{\mathrm{m}}^{*}$ ), as predicted by Eq. (35). On the other hand, for a spherical particle with a relatively high conductivity in a concentric cell with a constant temperature at the outer boundary as given by Eq. (7), the local temperature gradient at the particle surface will be enhanced in comparison with that for a corresponding isolated particle; thus, the value of the normalized photophoretic mobility $U / U^{(0)}$ increases with an increase in $k^{*}$.

\section{Concluding remarks}

Collections of particles are often encountered in real situations of photophoresis. It is important to understand if the effect of particle interactions significantly affects the movement of the particles. In this work, the photophoresis of a swarm of identical spherical particles suspended uniformly in a gaseous medium has been analyzed using the Happel and Kuwabara cell models with different boundary conditions at the virtual surface of the cell. Based on the assumption of small Knudsen, Peclet, and Reynolds numbers, the temperature and fluid flow fields in the cell are analytically solved and the particle velocities as functions of the volume fraction of the particles are obtained 
in the closed-form expressions of Eqs. (34) and (39). A comparison of the results between the two cell models has also been provided. We note that the two cell models lead to qualitatively the same but quantitatively somewhat different results of the mean particle velocity. This photophoretic velocity relative to its undisturbed value decreases monotonically with an increase in the particle concentration $\varphi$ and with a decrease in the relative conductivity $k^{*}$ of the particles. The relevant experimental data, which are not available in the literature, would be needed for confirming the validity of the cell models at various ranges of the parameters $k^{*}, C_{\mathrm{t}}^{*}, C_{\mathrm{m}}^{*}$, and $\varphi$.

It is worth repeating that Eqs. (34) and (39) are obtained on the basis of a continuum model for the gas phase with a slip-flow boundary condition at the particle surface. For a perfect gas, the kinetic theory predicts that the mean free path of gas molecules is inversely proportional to the pressure [23]. As examples, the mean free path of air molecules at $25{ }^{\circ} \mathrm{C}$ is approximately $67 \mathrm{~nm}$ at $1 \mathrm{~atm}$ and is approximately $51 \mu \mathrm{m}$ at 1 torr. Therefore, our results obtained with the assumption of small Knudsen number can be used for a broad range of particle sizes around atmospheric pressure but is only applicable for relatively large particles at low pressures.

\section{Acknowledgements}

Part of this work was supported by the National Science Council of the Republic of China.

\section{Appendix A. Nomenclature}

\begin{tabular}{|c|c|}
\hline$a$ & particle radius $(\mathrm{m})$ \\
\hline$A_{n}, B_{n}$ & coefficients in Eqs. (8) and (9) \\
\hline & radius of a unit cell $(\mathrm{m})$ \\
\hline$B(\zeta, \theta)$ & $\begin{array}{l}\text { dimensionless electric field dis- } \\
\text { tribution function defined by } \\
\text { Eq. (4) }\end{array}$ \\
\hline$C, D, E, F$ & $\begin{array}{l}\text { coefficients in Eq. (20) }\left(\mathrm{m}^{4}\right. \\
\left.\mathrm{s}^{-1}, \mathrm{~m}^{2} \mathrm{~s}^{-1}, \mathrm{~m} \mathrm{~s}^{-1}, \mathrm{~m}^{-1} \mathrm{~s}^{-1}\right)\end{array}$ \\
\hline$C_{\mathrm{m}}$ & $\begin{array}{l}\text { dimensionless coefficient ac- } \\
\text { counting for the frictional slip }\end{array}$ \\
\hline$C_{\mathrm{m}}^{*}$ & $=C_{\mathrm{m}} l / a$ \\
\hline
\end{tabular}

$C_{\mathrm{s}}$
$C_{\mathrm{t}}$
$C_{\mathrm{t}}^{*}$
$G$
$I$
$J_{1}$
$k$
$k_{\mathrm{p}}$
$k^{*}$
$l$
$r$
$S_{\mathrm{n}}(\zeta)$
$T$
$T_{\mathrm{p}}$
$T_{0}$
$U$

$\mathbf{v}$ $v_{\mathrm{r}}, v_{\theta}, v_{\phi}$

V

$z$

Greek letters

$\alpha, \beta$

$\zeta$

$\eta$

$\theta, \phi$

$\lambda$ dimensionless coefficient accounting for the thermal slip dimensionless coefficient accounting for the temperature jump

$=C_{\mathrm{t}} l / a$

parameter defined by Eq. (36) intensity of the incident light beam $\left(\mathrm{W} \mathrm{m}^{-2}\right)$

the photophoretic asymmetry factor of the particle thermal conductivity of the fluid $\left(\mathrm{W} \mathrm{m}^{-1} \mathrm{~K}^{-1}\right)$ thermal conductivity of the particle $\left(\mathrm{W} \mathrm{m}^{-1} \mathrm{~K}^{-1}\right)$ $=k_{\mathrm{p}} / k$

mean free path of the gas molecules (m) radial spherical coordinate $(\mathrm{m})$ function of $\zeta$ defined by Eq. (10) temperature distribution in the fluid phase $(\mathrm{K})$ temperature distribution inside the particle $(\mathrm{K})$ temperature of the bulk fluid (K) photophoretic velocity of a particle $\left(\mathrm{m} \mathrm{s}^{-1}\right)$ photophoretic velocity of an isolated particle $\left(\mathrm{m} \mathrm{s}^{-1}\right)$ fluid velocity field $\left(\mathrm{m}^{-1}\right)$ components of $v$ in spherical coordinates $\left(\mathrm{m} \mathrm{s}^{-1}\right)$ characteristic velocity defined by Eq. (23) $\left(\mathrm{m} \mathrm{s}^{-1}\right)$ coordinate in the direction of incident light (m)

coefficients defined by Eq.

(37)

$=r / a$

viscosity of the fluid $\left(\mathrm{kg} \mathrm{m}^{-1}\right.$ $\mathrm{s}^{-1}$ )

angular spherical coordinates the wavelength of the incident light beam (m) 

$v, \kappa$
real and imaginary parts of the complex refractive index of the particle
$\rho$
$\varphi$
$\Psi(r, \theta)$ density of the fluid $\left(\mathrm{kg} \mathrm{m}^{-3}\right)$ volume fraction of particles in a suspension $\left(=a^{3} / b^{3}\right)$
Stokes stream function of the fluid flow $\left(\mathrm{m}^{3} \mathrm{~s}^{-1}\right)$

\section{References}

[1] C. Orr, E.Y.H. Keng, J. Atmos. Sci. 21 (1964) 475.

[2] O. Preining, in: C.N. Davies (Ed.), Aerosol Science, Academic Press, New York, 1966, p. 111.

[3] J.C. Maxwell, Philos. Trans. R. Soc. 170 (1879) 231.

[4] E.H. Kennard, Kinetic Theory of Gases, McGraw-Hill, New York, 1938, p. 291.

[5] S. Arnold, M. Lewittes, J. Appl. Phys. 53 (1982) 5314.

[6] V. Chernyak, S. Beresnev, J. Aerosol Sci. 24 (1993) 857.

[7] M. Kerker, D.D. Cooke, J. Opt. Soc. Am. 72 (1982) 1267.
[8] D.W. Mackowski, Int. J. Heat Mass Transfer 32 (1989) 843.

[9] L.D. Reed, J. Aerosol Sci. 8 (1977) 123.

[10] Yu.I. Yalamov, V.B. Kutukov, E.R. Shchukin, J. Colloid Interface Sci. 57 (1976) 564.

[11] L. Talbot, R.K. Cheng, R.W. Schefer, D.R. Willis, J. Fluid Mech. 101 (1980) 737.

[12] J. Happel, AIChE J. 4 (1958) 197.

[13] S. Kuwabara, J. Phys. Soc. Jpn. 14 (1959) 527.

[14] S. Levine, G. Neale, N. Epstein, J. Colloid Interface Sci. 57 (1976) 424.

[15] H. Ohshima, J. Colloid Interface Sci. 208 (1998) 295.

[16] H.J. Keh, N.Y. Ho, J. Colloid Interface Sci. 216 (1999) 167.

[17] W.M. Greene, R.E. Spjut, E. Bar-Ziv, A.F. Sarofim, J.P. Longwell, J. Opt. Soc. Am. B 2 (1985) 998.

[18] J.R. Brock, J. Colloid Sci. 17 (1962) 768.

[19] J. Happel, H. Brenner, Low Reynolds Number Hydrodynamics, Martinus Nijhoff, The Netherlands, 1983.

[20] H.J. Keh, H.J. Tu, Colloids Surfaces A 176 (2001) 213.

[21] S. Levine, G.H. Neale, J. Colloid Interface Sci. 47 (1974) 520.

[22] H.J. Keh, S.H. Chen, Chem. Eng. Sci. 50 (1995) 3995.

[23] D.P. Shoemaker, C.W. Garland, J.I. Steinfeld, J.W Nibler, Experiments in Physical Chemistry, 4th ed., McGraw-Hill, New York, 1981. 